\title{
A CONSENSUS MODEL FOR GROUP DECISION MAKING PROBLEMS WITH UNBALANCED FUZZY LINGUISTIC INFORMATION
}

\author{
F.J. CABRERIZO \\ Department of Computer Science and Artificial Intelligence \\ University of Granada, Granada, 18071, Spain \\ cabrerizo@decsai.ugr.es \\ S. ALONSO \\ Department of Software Engineering \\ University of Granada, Granada, 18071, Spain \\ zerjioi@ugr.es \\ E. HERRERA-VIEDMA \\ Department of Computer Science and Artificial Intelligence \\ University of Granada, Granada, 18071, Spain \\ viedma@decsai.ugr.es
}

Received (Day Month Year)

Revised (Day Month Year)

\begin{abstract}
Most group decision making problems based on linguistic approaches use symmetrically and uniformly distributed linguistic term sets to express experts' opinions. However, there exist problems whose assessments need to be represented by means of unbalanced linguistic term sets, i.e., using term sets which are not uniformly and symmetrically distributed. The aim of this paper is to present a consensus model for group decision making problems with unbalanced fuzzy linguistic information. This consensus model is based on both a fuzzy linguistic methodology to deal with unbalanced linguistic term sets and two consensus criteria, consensus degrees and proximity measures. To do so, we use a new fuzzy linguistic methodology improving another approach to manage unbalanced fuzzy linguistic information, ${ }^{1}$ which uses the linguistic 2-tuple model as representation base of unbalanced fuzzy linguistic information. In addition, the consensus model presents a feedback mechanism to help experts for reaching the highest degree of consensus possible. There are two main advantages provided by this consensus model. Firstly, its ability to cope with group decision making problems with unbalanced fuzzy linguistic information overcoming the problem of finding different discrimination levels in linguistic term sets. And, secondly, it supports the consensus process automatically, avoiding the possible subjectivity that the moderator can introduce in this phase.
\end{abstract}

Keywords: Fuzzy linguistic modeling; unbalanced linguistic term set; group decision making; consensus. 


\section{Introduction}

It is undeniably true that Group Decision Making (GDM) problems arise from many real-world situations. ${ }^{2}$ In these problems, there are a set of alternatives to solve a problem and a group of experts characterized by their background and knowledge trying to achieve a common solution. To solve these problems, the experts are faced by applying two processes before obtaining a final solution: ${ }^{3,4,5,6,7,8}$ the consensus process and the selection process (see Fig. 1). The former consists in obtaining the maximum degree of consensus or agreement between the set of experts on the solution set of alternatives. Normally, the consensus process is guided by a human figure called moderator ${ }^{3,6}$ who is a person that does not participate in the discussion but monitors the agreement in each moment of the consensus process and is in charge of supervising and addressing the consensus process toward success, i.e., to achieve the maximum possible agreement and to reduce the number of experts outside of the consensus in each new consensus round. The latter refers to obtaining the solution set of alternatives from the opinions on the alternatives given by the experts. Clearly, it is preferable that the set of experts achieves a great agreement among their opinions before applying the selection process, and therefore, in this paper, we focus on the consensus process.

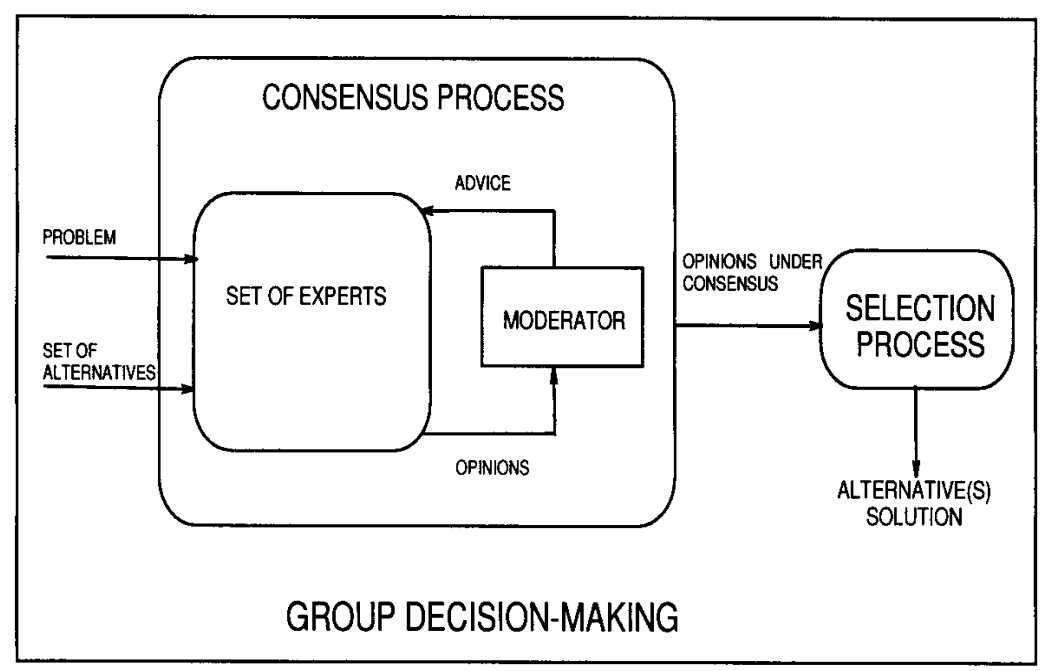

Fig. 1. Resolution process of a GDM problem

A consensus process is defined as a dynamic and iterative group discussion process, coordinated by a moderator helping experts bring their opinions closer. If the consensus level is lower than a specified threshold the moderator would urge experts to discuss their opinions further in an effort to bring them closer. On the contrary, 
when the consensus level is higher than the threshold, the moderator would apply the selection process in order to obtain the final consensus solution to the GDM problem. In this framework, an important question is how to substitute the actions of the moderator in the group discussion process in order to automatically model the whole consensus process. Some approaches have been proposed in GDM with multiple representation formats, ${ }^{4}$ in GDM with multi-granular linguistic information ${ }^{9}$ and in GDM with incomplete information. ${ }^{5,10}$

On the other hand, usually many problems present quantitative aspects which can be assessed by means of precise numerical values. ${ }^{2,7}$ However, some problems present also qualitative aspects that are complex to assess by means of precise and exact values. In these cases, the fuzzy linguistic approach $9,11,12,13,14,15,16,17,18,19,20$ can be used to obtain a better solution. This is the case, for instance, when experts try to evaluate the "comfort" of a car, where linguistic terms like "good", "fair", "poor" are used. ${ }^{21}$ Many of these problems use linguistic variables assessed in linguistic term sets whose terms are uniformly and symmetrically distributed, i.e., assuming the same discrimination levels on both sides of mid linguistic term. However, there exist problems that need to assess their variables with linguistic term sets that are not uniformly and symmetrically distributed. ${ }^{1,22}$ This type of linguistic term sets are called unbalanced linguistic term sets (see Fig. 2).

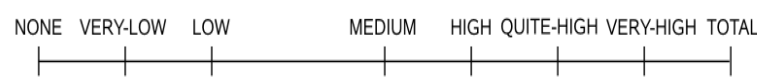

Fig. 2. Example of an unbalanced linguistic term set of 8 labels

The aim of this paper is to present a consensus model for GDM problems where the experts provide their opinions by means of unbalanced fuzzy linguistic information. In $^{1}$ a methodology to manage unbalanced fuzzy linguistic information in information retrieval systems was presented which used hierarchical linguistic contexts based on the linguistic 2-tuple computational model. ${ }^{23,24}$ However, this methodology can only represent unbalanced linguistic term sets when there exists a level with an adequate granularity to represent the subset of linguistic terms on the left of the mid linguistic term and a level with an adequate granularity to represent the subset of linguistic terms on the right of the mid linguistic term. Thus, we present a new fuzzy linguistic methodology that can represent unbalanced fuzzy linguistic information when the above conditions are not satisfied. As part of the consensus model, a feedback mechanism substituting the figure of the moderator is given to help experts change their opinions on the alternatives in order to obtain the highest degree of consensus possible. The feedback mechanism consists of simple and easy rules that generate recommendations in the discussion process. Moreover, this model is based on two types of consensus criteria, consensus degrees evaluating 
the agreement of all the experts and deciding when the consensus process should stop, and proximity measures evaluating the distance between experts' individual opinions and the group or collective opinion which is also used in the feedback mechanism to guide the direction of the changes in experts' opinions in order to increase the consensus degrees. Both measures are computed at the three different levels of representation of information of a preference relation: pair of alternatives, alternative and relation.

In order to do this, the paper is structured as follows. In Section 2, we present the new fuzzy linguistic methodology to manage unbalanced fuzzy linguistic information. Section 3 introduces the new consensus model for GDM problems with unbalanced fuzzy linguistic information. In Section 4, a practical example is given to illustrate the application of the consensus model. Finally, some concluding remarks are pointed out in Section 5 .

\section{A New Fuzzy Linguistic Methodology to Manage Unbalanced Fuzzy Linguistic Information}

In this section, we make a review of the 2-tuple fuzzy linguistic representation model ${ }^{23}$ and the concept of hierarchical linguistic contexts ${ }^{24}$ in order to present the new methodology to manage unbalanced fuzzy linguistic information.

\subsection{The 2-tuple fuzzy linguistic representation model}

The 2-tuple fuzzy linguistic representation model was introduced $i^{23}$ to carry out processes of computing with words $(\mathrm{CW})$ in a precise way when the linguistic term sets are symmetrically and uniformly distributed and to improve several aspects of the ordinal fuzzy linguistic approach. ${ }^{25,26,27,28}$ This model is based on the concept of symbolic translation and represents the linguistic information by means of a pair of values, $(s, \alpha)$, where $s$ is a linguistic label and $\alpha$ is a numerical value that represents the value of the symbolic translation.

Definition 2.1. ${ }^{23}$ Let $\beta \in[0, g]$ be the result of an aggregation of the indexes of a set of labels assessed in a linguistic term set $S=\left\{s_{0}, s_{1}, \ldots, s_{g-1}, s_{g}\right\}$, where $g$ stands for cardinality of $S$, i.e., the result of a symbolic aggregation operation. Let $i=\operatorname{round}(\beta)$ and $\alpha=\beta-i$ be two values, such that, $i \in[0, g]$ and $\alpha \in[-0.5,0.5)$, then $\alpha$ is called a symbolic translation.

This model defines a set of transformation functions to manage the linguistic information expressed by linguistic 2 -tuples.

Definition 2.2. Let $S$ be a linguistic term set and $\beta \in[0, g]$ a value supporting the result of a symbolic aggregation operation, then the 2 -tuple that expresses the 
equivalent information to $\beta$ is obtained with the following function:

$$
\begin{aligned}
\Delta: & {[0, g] \longrightarrow S \times[-0.5,0.5) } \\
& \Delta(\beta)=\left(s_{i}, \alpha\right) \\
& i=\operatorname{round}(\beta) \\
& \alpha=\beta-i
\end{aligned}
$$

where "round" is the usual round operation, $s_{i}$ has the closest index label to " $\beta$ " and " $\alpha$ " is the value of the symbolic translation.

Proposition 2.1. Let $S=\left\{s_{0}, \ldots, s_{g}\right\}$ be a linguistic term set and $\left(s_{i}, \alpha\right)$ be a linguistic 2-tuple. There is always a function $\Delta^{-1}$, such that, from a 2-tuple value it returns its equivalent numerical value $\beta \in[0, g] \subset \mathbb{R}$ :

$$
\begin{gathered}
\Delta^{-1}: S \times[-0.5,0.5) \longrightarrow[0, g] \\
\Delta^{-1}\left(s_{i}, \alpha\right)=i+\alpha=\beta
\end{gathered}
$$

Remark 2.1. We should point out that a linguistic term can be seen as a linguistic 2 -tuple by adding to it the value 0 as symbolic translation, $s_{i} \in S \Longrightarrow\left(s_{i}, 0\right)$.

The 2-tuples linguistic computational model presents different techniques to manage the linguistic information: ${ }^{23}$

(i) A 2-tuple comparison operator: The comparison of linguistic information represented by 2-tuples is carried out according to an ordinary lexicographic order. Let $\left(s_{k}, \alpha_{1}\right)$ and $\left(s_{l}, \alpha_{2}\right)$ be two 2 -tuples, with each one representing a counting of information:

(a) if $k<l$ then $\left(s_{k}, \alpha_{1}\right)$ is smaller than $\left(s_{l}, \alpha_{2}\right)$.

(b) if $k=l$ then

i. if $\alpha_{1}=\alpha_{2}$ then $\left(s_{k}, \alpha_{1}\right),\left(s_{l}, \alpha_{2}\right)$ represent the same information.

ii. if $\alpha_{1}<\alpha_{2}$ then $\left(s_{k}, \alpha_{1}\right)$ is smaller than $\left(s_{l}, \alpha_{2}\right)$.

iii. if $\alpha_{1}>\alpha_{2}$ then $\left(s_{k}, \alpha_{1}\right)$ is bigger than $\left(s_{l}, \alpha_{2}\right)$.

(ii) A 2-tuple negation operator: Is defined as

$$
N e g\left(s_{i}, \alpha\right)=\Delta\left(g-\Delta^{-1}\left(s_{i}, \alpha\right)\right) .
$$

(iii) 2-tuple aggregation operators: Using the function $\Delta$ and $\Delta^{-1}$ any aggregation operator can be easily extended for dealing with linguistic 2-tuples, such as the LOWA operator, ${ }^{25}$ the weighted average operator, the OWA operator, etc., $\left(\mathrm{see}^{23}\right)$.

\subsection{Hierarchical linguistic contexts}

$\mathrm{In}^{24}$ the hierarchical linguistic contexts were introduced to improve the precision of processes of computing with words in multi-granular linguistic contexts. ${ }^{29,30} \mathrm{In}$ this work, we use them to manage the unbalanced fuzzy linguistic information.

A Linguistic Hierarchy is a set of levels, where each level represents a linguistic term set with different granularity from the remaining levels of the hierarchy. Each 
level is denoted as $l(t, n(t))$, where $t$ is a number indicating the level of the hierarchy, and $n(t)$ is the cardinality of the linguistic term set of $t$. Moreover, we assume levels containing linguistic terms whose membership functions are triangular-shaped, uniformly and symmetrically distributed in $[0,1]$, and linguistic term sets having an odd value of granularity where the central label represents the value of indifference. A graphical example of a linguistic hierarchy is shown in Fig. 3.

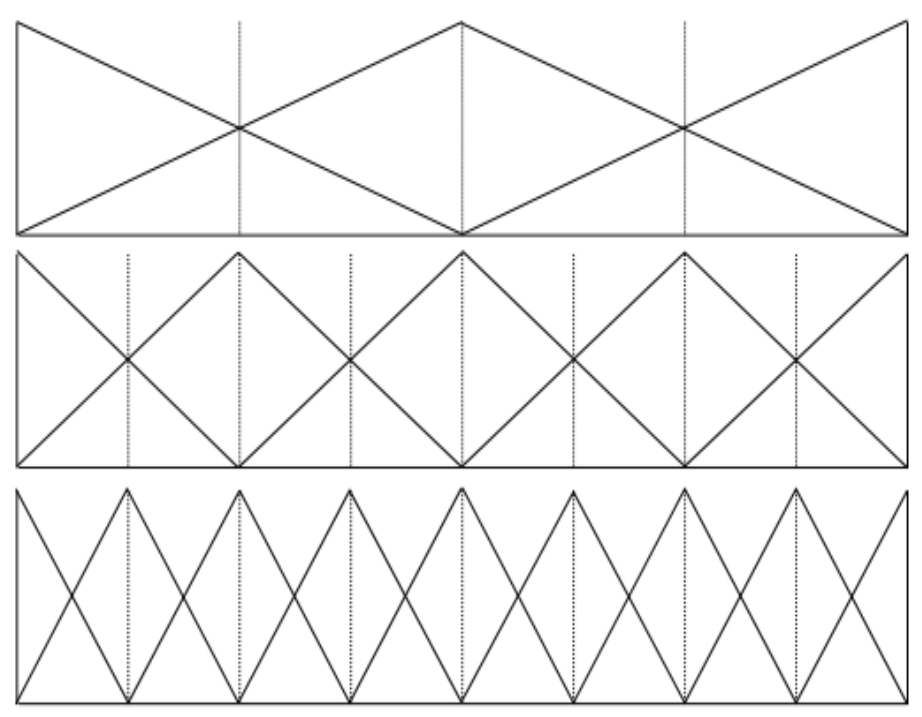

Fig. 3. Linguistic hierarchy of 3, 5 and 9 labels

The levels belonging to a linguistic hierarchy are ordered according to their granularity, i.e., for two consecutive levels $t$ and $t+1, n(t+1)>n(t)$. Hence, the level $t+1$ could be considered as a refinement of the previous level $t$. Then, a linguistic hierarchy $L H$ can be defined as the union of all levels $t$ :

$$
L H=\bigcup_{t} l(t, n(t)) .
$$

Given a $L H$, we denote as $\mathcal{S}^{n(t)}$ the linguistic term set of $L H$ corresponding to the level $t$ of $L H$ characterized by a granularity of uncertainty $n(t): \mathcal{S}^{n(t)}=$ $\left\{s_{0}^{n(t)}, \ldots, s_{n(t)-1}^{n(t)}\right\}$. Furthermore, the linguistic term set of the level $t+1$ is obtained from its predecessor as:

$$
l(t, n(t)) \rightarrow l(t+1,2 \cdot n(t)-1) .
$$

Transformation functions between labels from different levels to make processes of computing with words in multigranular linguistic information contexts without 
loss of information were defined in. ${ }^{24}$

Definition 2.3. ${ }^{24}$ Let $L H=\bigcup_{t} l(t, n(t))$ be a linguistic hierarchy whose linguistic term sets are denoted as $\mathcal{S}^{n(t)}=\left\{s_{0}^{n(t)}, \ldots, s_{n(t)-1}^{n(t)}\right\}$, and let us consider the 2-tuple fuzzy linguistic representation. The transformation function from a linguistic label in level $t$ to a label in level $t^{\prime}$ is defined as $T F_{t^{\prime}}^{t}: l(t, n(t)) \longrightarrow l\left(t^{\prime}, n\left(t^{\prime}\right)\right)$ such that

$$
T F_{t^{\prime}}^{t}\left(s_{i}^{n(t)}, \alpha^{n(t)}\right)=\Delta_{t^{\prime}}\left(\frac{\Delta_{t}^{-1}\left(s_{i}^{n(t)}, \alpha^{n(t)}\right) \cdot\left(n\left(t^{\prime}\right)-1\right)}{n(t)-1}\right) .
$$

\subsection{A new model to manage unbalanced fuzzy linguistic information}

A new model to manage unbalanced fuzzy linguistic term sets based on the linguistic 2-tuple model is presented. It carries out computational operations of unbalanced fuzzy linguistic information using the 2-tuple computational model and different levels of a $L H$. This new model presents two components:

- a representation model of unbalanced fuzzy linguistic information and

- a computational model of unbalanced fuzzy linguistic information.

As aforementioned, this methodology overcomes the problem of that model proposed in $^{1}$ because it represents unbalanced fuzzy linguistic information when there does not exist a level in a $L H$ with an adequate granularity to represent the subset of linguistic terms on the left of the mid linguistic term or on the right of the mid linguistic term.

\subsubsection{An unbalanced fuzzy linguistic representation model}

The procedure to represent unbalanced fuzzy linguistic information defined in ${ }^{1}$ works as follows:

(i) Find a level $t^{-}$of $L H$ to represent the subset of linguistic terms $\mathcal{S}_{u n}^{L}$ on the left of the mid linguistic term of unbalanced fuzzy linguistic term set $\mathcal{S}_{\text {un }}$. This level of $L H$ should support the distribution of the labels of $\mathcal{S}_{u n}^{L}$ on the discourse universe.

(ii) Find a level $t^{+}$of $L H$ to represent the subset of linguistic terms $\mathcal{S}_{u n}^{R}$ on the right of the mid linguistic term of $\mathcal{S}_{\text {un }}$.

(iii) Represent the mid term of $\mathcal{S}_{\text {un }}$ using the mid terms of the levels $t^{-}$and $t^{+}$.

As aforementioned, the problem appears when there does not exist a level $t^{-}$or $t^{+}$in $L H$ to represent $\mathcal{S}_{u n}^{L}$ or $\mathcal{S}_{u n}^{R}$, respectively. Then, we propose to overcome this problem by applying the following algorithm, which is defined assuming that there does not exist $t^{-}$, as it happens with the unbalanced fuzzy linguistic term set given in Fig. 2:

(i) Represent $\mathcal{S}_{\text {un }}^{L}$ : 
(a) Identify the mid term of $\mathcal{S}_{\text {un }}^{L}$, called $\mathcal{S}_{\text {mid }}^{L}$. To do so, we have to observe the distribution of the labels of $\mathcal{S}_{u n}^{L}$ on the discourse universe.

(b) Find a level $t_{2}^{-}$of the left sets of $L H^{L}$ to represent the left term subset of $\mathcal{S}_{u n}^{L}$, where $L H^{L}$ represents the left part of $L H$.

(c) Find a level $t_{2}^{+}$of the right sets of $L H^{L}$ to represent the right term subset of $\mathcal{S}_{u n}^{L}$.

(d) Represent the mid term $\mathcal{S}_{m i d}^{L}$ using the levels $t_{2}^{-}$and $t_{2}^{+}$.

(ii) Find a level $t^{+}$of $L H$ to represent the subset of linguistic terms $\mathcal{S}_{u n}^{R}$.

(iii) Represent the mid term of $\mathcal{S}_{\text {un }}$ using the levels $t^{+}$and $t_{2}^{+}$.

For example, applying this algorithm, the representation of the unbalanced fuzzy linguistic term set $\mathcal{S}_{u n}=\{N, V L, L, M, H, Q H, V H, T\}$ shown in Fig. 2 with the linguistic hierarchy $L H$ shown in Fig. 3 would be as it is shown in Fig. 4 . In this example,

- $\mathcal{S}_{u n}^{L}=\{N, V L, L\}$,

- $\mathcal{S}_{\text {mid }}^{L}=L$,

- $L H^{L}=\left\{s_{0}^{n(1)}\right\} \bigcup\left\{s_{0}^{n(2)}, s_{1}^{n(2)}\right\} \bigcup\left\{s_{0}^{n(3)}, s_{1}^{n(3)}, s_{2}^{n(3)}, s_{3}^{n(3)}\right\}$.

Thus, we have that $t_{2}^{-}=3, t_{2}^{+}=2$, the mid label $\mathcal{S}_{m i d}^{L}=L$ (due to its position on the discourse universe) is represented using both levels, 3 and 2 , and the mid term of $\mathcal{S}_{\text {un }}$ is represented using the levels 2 and 3.

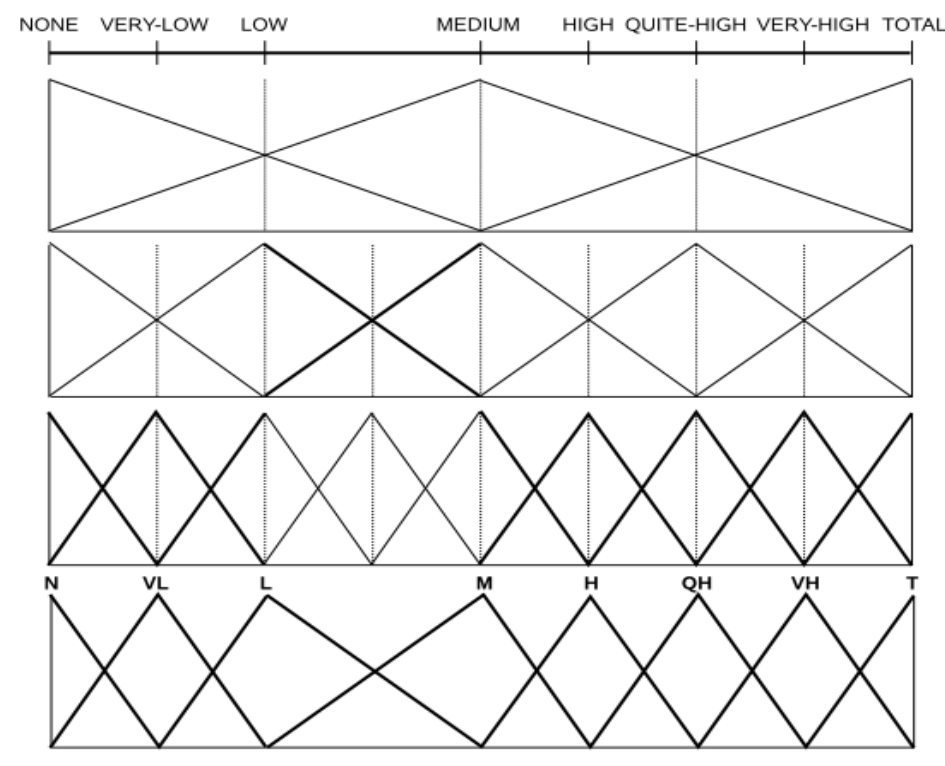

Fig. 4. Representation for an unbalanced term set of 8 labels 


\subsubsection{An unbalanced fuzzy linguistic computational model}

In any fuzzy linguistic approach we need to define a computational model to manage and aggregate linguistic information. As in, ${ }^{23,26}$ we have to define three types of computation operators to deal with unbalanced fuzzy linguistic information: comparison operators, negation operator and aggregation operators. In an unbalanced linguistic context, previously to carry out any computation task of unbalanced fuzzy linguistic information we have to choose a level $t^{\prime} \in\left\{t^{-}, t_{2}^{-}, t^{+}, t_{2}^{+}\right\}$, such that $n\left(t^{\prime}\right)=\max \left\{n\left(t^{-}\right), n\left(t_{2}^{-}\right), n\left(t^{+}\right), n\left(t_{2}^{+}\right)\right\}:$

(i) An unbalanced linguistic comparison operator: The comparison of linguistic information represented by two unbalanced linguistic 2-tuples $\left(s_{k}^{n(t)}, \alpha_{1}\right)$, $t \in\left\{t^{-}, t_{2}^{-}, t^{+}, t_{2}^{+}\right\}$, and $\left(s_{l}^{n(t)}, \alpha_{2}\right), t \in\left\{t^{-}, t_{2}^{-}, t^{+}, t_{2}^{+}\right\}$is similar to the usual comparison of two 2-tuples but acting on the values $T F_{t^{\prime}}^{t}\left(s_{k}^{n(t)}, \alpha_{1}\right)=\left(s_{v}^{n\left(t^{\prime}\right)}, \beta_{1}\right)$ and $T F_{t^{\prime}}^{t}\left(s_{l}^{n(t)}, \alpha_{2}\right)=\left(s_{w}^{n\left(t^{\prime}\right)}, \beta_{2}\right)$. Then, we have:

(a) if $v<w$ then $\left(s_{v}^{n\left(t^{\prime}\right)}, \beta_{1}\right)$ is smaller than $\left(s_{w}^{n\left(t^{\prime}\right)}, \beta_{2}\right)$.

(b) if $v=w$ then

i. if $\beta_{1}=\beta_{2}$ then $\left(s_{v}^{n\left(t^{\prime}\right)}, \beta_{1}\right),\left(s_{w}^{n\left(t^{\prime}\right)}, \beta_{2}\right)$ represent the same information.

ii. if $\beta_{1}<\beta_{2}$ then $\left(s_{v}^{n\left(t^{\prime}\right)}, \beta_{1}\right)$ is smaller than $\left(s_{w}^{n\left(t^{\prime}\right)}, \beta_{2}\right)$.

iii. if $\beta_{1}>\beta_{2}$ then $\left(s_{v}^{n\left(t^{\prime}\right)}, \beta_{1}\right)$ is bigger than $\left(s_{w}^{n\left(t^{\prime}\right)}, \beta_{2}\right)$.

(ii) An unbalanced linguistic 2-tuple negation operator. Let $\left(s_{k}^{n(t)}, \alpha\right), \quad t \in$ $\left\{t^{-}, t_{2}^{-}, t^{+}, t_{2}^{+}\right\}$be an unbalanced linguistic 2-tuple, then:

$$
\mathcal{N} \mathcal{E} \mathcal{G}\left(s_{k}^{n(t)}, \alpha\right)=\operatorname{Neg}\left(T F_{t^{\prime \prime}}^{t}\left(s_{k}^{n(t)}, \alpha\right)\right),
$$

where $t \neq t^{\prime \prime}, t^{\prime \prime} \in\left\{t^{-}, t_{2}^{-}, t^{+}, t_{2}^{+}\right\}$.

(iii) An unbalanced linguistic aggregation operator. As aforementioned, in order to deal with unbalanced fuzzy linguistic information we have to represent it in a $L H$. Hence, any unbalanced linguistic aggregation operator must aggregate unbalanced fuzzy linguistic information by means of its representation in a $L H$. We use the aggregation processes designed in the 2-tuple computational model but acting on the unbalanced linguistic values transformed by means of $T F_{t^{\prime}}^{t}$. Then, once a result is obtained, it is transformed to the correspondent level $t \in\left\{t^{-}, t_{2}^{-}, t^{+}, t_{2}^{+}\right\}$by means of $T F_{t}^{t^{\prime}}$ for expressing the result in the unbalanced linguistic term set $\mathcal{S}_{\text {un }}$. In this way, the $L O W A_{\text {un }}$ operator, which is an extension of the Linguistic Ordered Weighted Averaging operator, ${ }^{25}$ is defined as follows:

Definition 2.4. Let $\left\{\left(a_{1}, \alpha_{1}\right), \ldots,\left(a_{m}, \alpha_{m}\right)\right\}$ be a set of unbalanced assessments to aggregate, then the $L O W A_{u n}$ operator $\phi_{u n}$ is defined as:

$$
\begin{gathered}
\phi_{u n}\left\{\left(a_{1}, \alpha_{1}\right), \ldots,\left(a_{m}, \alpha_{m}\right)\right\}=W \cdot B^{T}=C_{u n}^{m}\left\{w_{k}, b_{k}, k=1, \ldots, m\right\}= \\
w_{1} \otimes b_{1} \oplus\left(1-w_{1}\right) \otimes C_{u n}^{m-1}\left\{\beta_{h}, b_{h}, h=2, \ldots, m\right\}
\end{gathered}
$$


where $b_{i}=\left(a_{i}, \alpha_{i}\right) \in\left(\mathcal{S}_{\text {un }} \times[-0.5,0.5)\right), W=\left[w_{1}, \ldots, w_{m}\right]$, is a weighting vector, such that, $w_{i} \in[0,1]$ and $\sum_{i} w_{i}=1, \beta_{h}=\frac{w_{h}}{\sum_{2}^{m} w_{k}}, h=2, \ldots, m$, and $B$ is the associated ordered unbalanced 2-tuple vector. Each element $b_{i} \in B$ is the i-th largest unbalanced 2-tuple in the collection $\left\{\left(a_{1}, \alpha_{1}\right), \ldots,\left(a_{m}, \alpha_{m}\right)\right\}$, and $C_{u n}^{m}$ is the convex combination operator of $m$ unbalanced 2-tuples. If $w_{j}=1$ and $w_{i}=0$ with $i \neq j \forall i, j$ the convex combination is defined as: $C_{u n}^{m}\left\{w_{i}, b_{i}, i=\right.$ $1, \ldots, m\}=b_{j}$. And if $m=2$ then it is defined as:

$$
C_{u n}^{2}\left\{w_{l}, b_{l}, l=1,2\right\}=w_{1} \otimes b_{j} \oplus\left(1-w_{1}\right) \otimes b_{i}=T F_{t}^{t^{\prime}}\left(s_{k}^{n\left(t^{\prime}\right)}, \alpha\right)
$$

where $\left(s_{k}^{n\left(t^{\prime}\right)}, \alpha\right)=\Delta(\lambda)$ and $\lambda=\Delta^{-1}\left(T F_{t^{\prime}}^{t}\left(b_{i}\right)\right)+w_{1} \cdot\left(\Delta^{-1}\left(T F_{t^{\prime}}^{t}\left(b_{j}\right)\right)-\right.$ $\left.\Delta^{-1}\left(T F_{t^{\prime}}^{t}\left(b_{i}\right)\right)\right), b_{j}, b_{i} \in\left(\mathcal{S}_{u n} \times[-0.5,0.5)\right),\left(b_{j} \geq b_{i}\right), \lambda \in\left[0, n\left(t^{\prime}\right)-1\right], t \in$ $\left\{t^{-}, t_{2}^{-}, t^{+}, t_{2}^{+}\right\}$.

$\mathrm{In}^{31}$ it was defined an expression to obtain $W$ by means of a fuzzy linguistic non-decreasing quantifier $Q:^{32}$

$$
w_{i}=Q(i / m)-Q((i-1) / m), \quad i=1, \ldots, m .
$$

\section{A Consensus Model for GDM Problems with Unbalanced Fuzzy Linguistic Information}

In this section, we present a consensus model defined for GDM problems with unbalanced fuzzy linguistic preference relations providing support to the experts to reach consensus during the process of making a decision. This consensus model presents the following main characteristics:

(i) It is designed to guide the consensus process of unbalanced fuzzy linguistic GDM problems.

(ii) It uses a methodology to manage unbalanced fuzzy linguistic information.

(iii) It is based on two consensus criteria: consensus degrees and proximity measures. The first ones are used to measure the agreement amongst all the experts, while the second ones are used to learn how close the collective and individual expert's preference are. Both consensus criteria are calculated at three different levels: pair of alternatives, alternatives and relation.

(iv) A feedback mechanism is defined using the above consensus criteria. It substitutes the moderator's actions, avoiding the possible subjectivity that he/she can introduce, and gives advice to the experts to find out the changes they need to make in their opinions in order to obtain the highest degree of consensus possible.

Although the main purpose of our consensus model is to support the experts throughout the consensus process, they are who decide whether or not to follow the advice generated by the consensus model. In any case, in our consensus model the time associated with making the decision is reduced and, therefore, it extends the experts' ability to analyze the information involved in the decision making process. 
In particular, our consensus model presents three phases that will be described in further detail in the following subsections:

(i) Computing consensus degrees. In this phase, the consensus degrees amongst the experts are calculated. To do so, a similarity measure is defined to obtain the coincidence amongst experts' opinions.

(ii) Controlling the consensus state. In this phase, the level of consensus and the number of rounds of discussion to be carried out is checked. In this way, if the agreement amongst the experts satisfies a minimum consensus threshold $(\gamma)$, the consensus process will stop and the selection process will be applied in order to obtain the solution set of alternatives. Otherwise, the third phase is carried out, i.e., the experts' opinions must be modified in order to increase the agreement amongst the experts. To avoid that the consensus process does not converge after several rounds of discussion, a maximum number of consensus rounds, MaxRounds, is incorporated.

(iii) Feedback mechanism. To help experts change their opinions, recommendations are provided by means of easy rules. To do this, proximity measures are used in conjunction with the consensus degrees to generate advice so that experts can change their opinions.

\subsection{Computing consensus degrees}

A GDM problem based on preference relations is classically defined as a decision situation where there are a set of experts, $E=\left\{e_{1}, \ldots, e_{m}\right\}(m \geq 2)$, and a finite set of alternatives, $X=\left\{x_{1}, \ldots, x_{n}\right\}(n \geq 2)$, and each expert $e_{i}$ provides his/her preferences about $X$ by means of a preference relation, $\mathbf{P}_{\mathbf{e}_{\mathbf{i}}} \subset X \times X$, where the value $\mu_{\mathbf{P}_{\mathbf{e}_{\mathbf{i}}}}\left(x_{j}, x_{k}\right)=p_{i}^{j k}$ is interpreted as the preference degree of the alternative $x_{j}$ over $x_{k}$ for $e_{i}$. In this paper, we deal with unbalanced fuzzy linguistic GDM problems, i.e., GDM problems where the experts $e_{i}$ express their preferences relations $\mathbf{P}_{\mathbf{e}_{\mathbf{i}}}=\left(p_{i}^{j k}\right)$ on the set of alternatives $X$ using a linguistic term set that is not uniformly and symmetrically distributed, $\mathcal{S}_{u n}$, which has a minimum label, a maximum label, a central label and the remaining labels are non-uniformly and non-symmetrically distributed around the central one, on both left and right lateral sets (as it happens, for example, in the grading system (Fig. 5)). Therefore, $p_{i}^{j k} \in \mathcal{S}_{\text {un }}$ represents the preference of alternative $x_{j}$ over alternative $x_{k}$ for the experts $e_{i}$ assessed on the unbalanced fuzzy linguistic term set $\mathcal{S}_{u n}$.

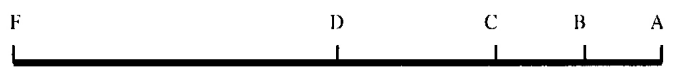

Fig. 5. Grading system evaluations

Then, consensus degrees are used to measure the current level of consensus in 
the decision process. As aforementioned, they are given at three different levels: pairs of alternatives, alternatives and relations. To calculate them, some similarity or coincidence function are required to obtain the level of agreement amongst all the experts. ${ }^{3,9,33}$ Moreover, these similarity functions detect how far each individual expert is from the rest. In this way, the computation of the consensus degrees is carried out as follows:

(i) For each pair of experts $\left(e_{i}, e_{j}\right)(i=1, \ldots, m-1, j=i+1, \ldots, m)$, a similarity matrix, $S M_{i j}=\left(s m_{i j}^{l k}\right)$, is defined where

$$
s m_{i j}^{l k}=1-\frac{\left|\Delta_{t^{\prime}}^{-1}\left(T F_{t^{\prime}}^{t}\left(p_{i}^{l k}\right)\right)-\Delta_{t^{\prime}}^{-1}\left(T F_{t^{\prime}}^{t}\left(p_{j}^{l k}\right)\right)\right|}{n\left(t^{\prime}\right)-1} .
$$

being $p_{i}^{l k}=\left(s_{v}^{n(t)}, \alpha_{1}\right), t \in\left\{t^{-}, t_{2}^{-}, t^{+}, t_{2}^{+}\right\}, p_{j}^{l k}=\left(s_{w}^{n(t)}, \alpha_{2}\right), t \in\left\{t^{-}, t_{2}^{-}, t^{+}, t_{2}^{+}\right\}$, and $t^{\prime} \in\left\{t^{-}, t_{2}^{-}, t^{+}, t_{2}^{+}\right\}$.

(ii) A consensus matrix, $C M=\left(\mathrm{cm}^{l k}\right)$, is calculated by aggregating all the similarity matrices using the arithmetic mean as the aggregation function $\phi$ :

$$
c m^{l k}=\phi\left(s m_{i j}^{l k}, i=1, \ldots, m-1, j=i+1, \ldots, m\right) .
$$

(iii) Once the consensus matrix, $C M$, is computed, we proceed to calculate the consensus degrees at the three different levels:

(a) Level 1. Consensus degree on pairs of alternatives. The consensus degree on a pair of alternatives $\left(x_{l}, x_{k}\right)$, called $c p^{l k}$, is defined to measure the consensus degree amongst all the experts on that pair of alternatives. The closer $c p^{l k}$ to 1 , the greater the agreement amongst all the experts on the pair of alternatives $\left(x_{l}, x_{k}\right)$. Thus, this measure is used to identify those pairs of alternatives with a poor level of consensus and is expressed by the element $(l, k)$ of the consensus matrix $C M$ :

$$
c p^{l k}=c m^{l k} ; \forall l, k=1, \ldots, n \wedge l \neq k .
$$

(b) Level 2. Consensus degree on alternatives. The consensus degree on an alternative $x_{l}$, called $c a^{l}$, is defined to measure the consensus degree amongst all the experts on that alternative:

$$
c a^{l}=\frac{\sum_{k=1}^{n} c p^{l k}}{n} .
$$

(c) Level 3. Consensus degree on the relation. The consensus degree on the relation, called $\mathrm{cr}$, is defined to measure the global consensus degree amongst all the experts' opinions and is used by the consensus model to control the consensus situation. It is calculated as the average of all the consensus degrees on the alternatives:

$$
c r=\frac{\sum_{l=1}^{n} c a^{l}}{n}
$$




\subsection{Controlling the consensus state}

The consensus state control process involves deciding if the feedback mechanism should be applied to provide advice to the experts or if the consensus process should be finished. To do so, a minimum consensus threshold, $\gamma \in[0,1]$, is fixed before applying the consensus model. It will depend on the particular problem we are dealing with. On the one hand, when the consequences of the decision to be made are of a transcendent importance, the minimum level of consensus required to make that decision should be logically as high as possible, and it is not unusual if a minimum value of 0.8 or higher is imposed. On the other hand, if the consequences are not so transcendental (but are still important) or it is urgent to obtain a solution to the problem, a minimum consensus value as close as possible to 0.5 could be required.

In any case, when the consensus measure, $c r$, satisfies the minimum consensus threshold, $\gamma$, the consensus model finishes and the selection process is applied to obtain the solution. Additionally, the consensus model should avoid situations in which the global consensus measure may not converge to the minimum consensus threshold. To do that, a maximum number of rounds MaxRounds should be fixed and compared to the current number of round of the consensus model NumRound.

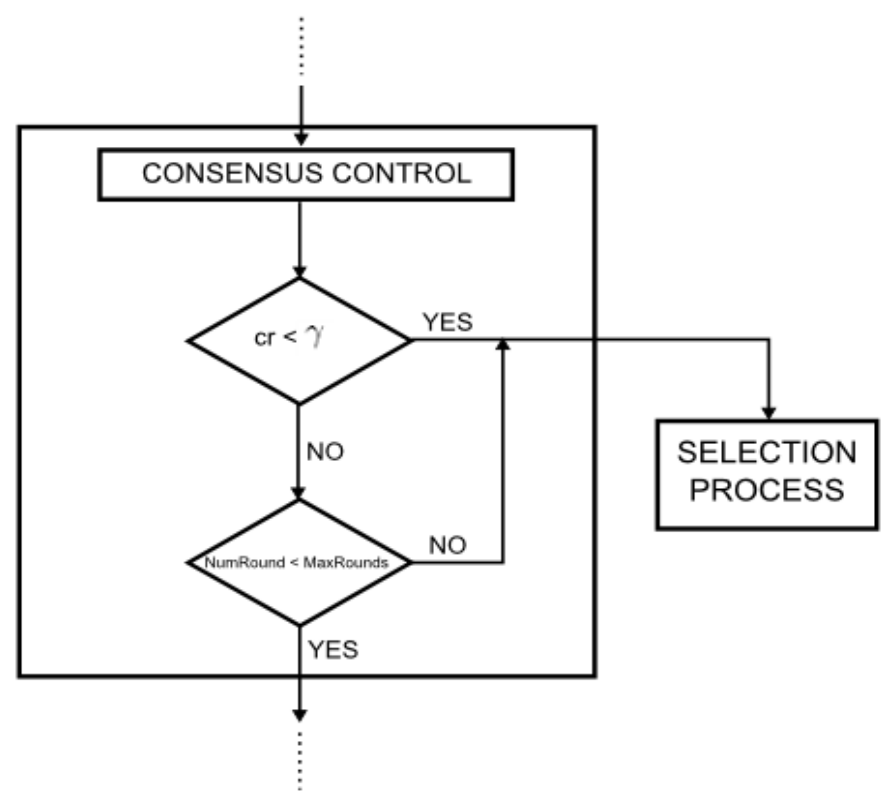

Fig. 6. Consensus control

In this way, the operation of the consensus state control process is the following (see Fig. 6): firstly, the global consensus measure, $c r$, is checked against the minimum 
consensus threshold, $\gamma$. If $\mathrm{cr}>\gamma$, the consensus process finishes and the selection process is applied. Otherwise, it will check if the maximum number of rounds, MaxRounds, has been reached. If so, it finishes and the selection process is applied too, and if not, it activates the feedback mechanism.

\subsection{Feedback mechanism}

As aforementioned, if the global consensus measure is lower than the minimum consensus threshold, the experts' opinions must be modified. This is done by the feedback mechanism which provides recommendations to support the experts in changing their opinions. To do so, the feedback mechanism uses proximity measures to identify those experts furthest away from the collective opinion. In the following, both the computation of the proximity measures and the production of advice are explained in detail.

\subsubsection{Computation of proximity measures}

These measures evaluate the agreement between the individual experts' opinions and the group opinion. To compute them for each expert, we need to obtain the collective unbalanced fuzzy linguistic preference relation, $\mathbf{P}_{\mathbf{e}_{\mathbf{c}}}=\left(p_{c}^{l k}\right)$, which summarizes preferences given by all the experts and is calculated by means of the aggregation of the set of individual unbalanced fuzzy linguistic preference relations $\left\{\mathbf{P}_{\mathbf{e}_{1}}, \ldots, \mathbf{P}_{\mathbf{e}_{\mathbf{m}}}\right\}$ as follows

$$
p_{c}^{l k}=\phi_{u n}\left(p_{1}^{l k}, \ldots, p_{m}^{l k}\right) .
$$

with $\phi_{u n}$ the $L O W A_{u n}$ operator defined in Section 2.3.

Once $\mathbf{P}_{\mathbf{e}_{\mathbf{c}}}$ is obtain, we can compute the proximity measures carrying out the following two steps:

(i) For each expert, $e_{i}$, a proximity matrix, $P M_{i}=\left(p m_{i}^{l k}\right)$, is obtained where

$$
p m_{i}^{l k}=1-\frac{\left|\Delta_{t^{\prime}}^{-1}\left(T F_{t^{\prime}}^{t}\left(p_{i}^{l k}\right)\right)-\Delta_{t^{\prime}}^{-1}\left(T F_{t^{\prime}}^{t}\left(p_{c}^{l k}\right)\right)\right|}{n\left(t^{\prime}\right)-1} .
$$

being $p_{i}^{l k}=\left(s_{v}^{n(t)}, \alpha_{1}\right), t \in\left\{t^{-}, t_{2}^{-}, t^{+}, t_{2}^{+}\right\}, p_{c}^{l k}=\left(s_{w}^{n(t)}, \alpha_{2}\right), t \in\left\{t^{-}, t_{2}^{-}, t^{+}, t_{2}^{+}\right\}$, and $t^{\prime} \in\left\{t^{-}, t_{2}^{-}, t^{+}, t_{2}^{+}\right\}$.

(ii) Computation of proximity measures at three different levels:

(a) Level 1. Proximity measure on pairs of alternatives. The proximity measure of an expert $e_{i}$ on a pair of alternatives $\left(x_{l}, x_{k}\right)$ to the group's one, called $p p_{i}^{l k}$, is expressed by the element $(l, k)$ of the proximity matrix $P M_{i}$ :

$$
p p_{i}^{l k}=p m_{i}^{l k} ; \forall l, k=1, \ldots, n \wedge l \neq k .
$$

(b) Level 2. Proximity measure on alternatives. The proximity measure of an expert $e_{i}$ on an alternative $x_{l}$ to the group's one, called $p a_{i}^{l}$, is calculated 
as follows:

$$
p a_{i}^{l}=\frac{\sum_{k=1}^{n} p p_{i}^{l k}}{n} .
$$

(c) Level 3. Proximity measure on the relation. The proximity measure of an expert $e_{i}$ on his/her unbalanced fuzzy linguistic preference relation to the group's one, called $p r_{i}$, is calculated as the average of all proximity measures on the alternatives:

$$
p r_{i}=\frac{\sum_{l=1}^{n} p a_{i}^{l}}{n}
$$

The meaning of the proximity measures are the following: if they are close to 1 , then they have a positive contribution for the consensus to be high, while if they are close to 0 , then they have a negative contribution to the consensus. Therefore, we can use them to provide advice to the experts to change their opinions and to find out which direction that change has to follow in order to obtain the highest degree of consensus possible.

\subsubsection{Production of advice}

The production of advice to achieve a solution with the highest degree of consensus possible is carried out in two steps: Identification rules and Direction rules.

(i) Identification rules (IR). We must identify the experts, alternatives and pairs of alternatives that are contributing less to reach a high degree of consensus and, therefore, should participate in the change process.

(a) Identification rule of experts (IR.1). It identifies the set of experts that should receive advice on how to change some of their preference values. This set of experts, called $E X P C H$, that should change their opinions are those whose proximity measure on the relation, $p r_{i}$, is lower than the minimum consensus threshold $\gamma$. Therefore, the identification rule of experts, IR.1, is the following:

$$
E X P C H=\left\{i \mid p r_{i}<\gamma\right\}
$$

(b) Identification rule of alternatives (IR.2). It identifies the alternatives whose associated assessments should be taken into account by the above experts in the change process of their preferences. This set of alternatives is denoted as $A L T_{i}$. The identification rule of alternatives, IR.2, is the following:

$$
A L T_{i}=\left\{x_{l} \in X \mid c a^{l}<\gamma \wedge e_{i} \in E X P C H\right\}
$$

(c) Identification rule of pairs of alternatives (IR.3). It identifies the particular pairs of alternatives $\left(x_{l}, x_{k}\right)$ whose respective associated assessments $p_{i}^{l k}$ the expert $e_{i}$ should change. This set of pairs of alternatives is denoted as $P A L T_{i}$. The identification rule of pairs of alternatives, IR.3, is the following:

$$
P A L T_{i}=\left\{\left(x_{l}, x_{k}\right) \mid x_{l} \in A L T \wedge e_{i} \in E X P C H \wedge p p_{i}^{l k}<\gamma\right\}
$$


(ii) Direction rules (DR). We must find out the direction of the change to be recommended in each case, i.e., the direction of change to be applied to the preference assessment $p_{i}^{l k}$, with $\left(x_{l}, x_{k}\right) \in P A L T_{i}$. To do this, we define the following two direction rules.

(a) DR.1. If $p_{i}^{l k}>p_{c}^{l k}$, the expert $e_{i}$ should decrease the assessment associated to the pair of alternatives $\left(x_{l}, x_{k}\right)$, i.e., $p_{i}^{l k}$.

(b) DR.2. If $p_{i}^{l k}<p_{c}^{l k}$, the expert $e_{i}$ should increase the assessment associated to the pair of alternatives $\left(x_{l}, x_{k}\right)$, i.e., $p_{i}^{l k}$.

Remark 3.1. These direction rules will not be produced when a decrease or increase are suggested to an assessment represented by the first or last label of the unbalanced linguistic term set, respectively.

Obviously, the consensus reaching process will depend on the size of the group of experts as well as on the size of the set of alternatives, so that when sizes are small and when opinions are homogeneous, the consensus level required is easier to obtain. ${ }^{4,34}$ On the other hand, we note that changes in the experts' opinions will produce a change in the collective opinion, specially when the experts opinions are quite different, i.e., in the early stages of the consensus process. In fact, when experts opinions are close, i.e., when the consensus measure approaches the consensus level required, changes in experts' opinions will not produce a great difference in the collective opinion.

\section{Example of Application}

For the sake of simplicity, we will assume a low number of experts and alternatives. Let us suppose that four different experts $E=\left\{e_{1}, e_{2}, e_{3}, e_{4}\right\}$ provide the following unbalanced fuzzy linguistic preference relations over a set of four alternatives $X=\left\{x_{1}, x_{2}, x_{3}, x_{4}\right\}$ using the unbalanced linguistic term set $\mathcal{S}_{u n}=\{N, V L, L, M, H, Q H, V H, T\}$ (see Fig. 2 and Fig. 4):

$$
\begin{aligned}
P_{e_{1}} & =\left(\begin{array}{cccc}
- & H & Q H & L \\
V L & - & M & H \\
L & M & - & L \\
V H & V L & V H & -
\end{array}\right) ; P_{e_{2}}=\left(\begin{array}{cccc}
- & V L & L & V H \\
H & - & Q H & T \\
Q H & L & - & H \\
V L & N & L & -
\end{array}\right) \\
P_{e_{3}} & =\left(\begin{array}{cccc}
-V L & M & V H \\
H & - & Q H & L \\
M & L & - & T \\
L & H & N & -
\end{array}\right) ; P_{e_{4}}=\left(\begin{array}{cccc}
- & V H & Q H & M \\
V L & - & M & V H \\
L & M & - & V L \\
M & L & Q H & -
\end{array}\right)
\end{aligned}
$$

Note that the expert did not provide any $\alpha$ values, which is a common practice when expressing preferences with linguistic terms. In these cases, we set $\alpha=0$.

$$
P_{e_{1}}=\left(\begin{array}{cccc}
- & (H, 0) & (Q H, 0) & (L, 0) \\
(V L, 0) & - & (M, 0) & (H, 0) \\
(L, 0) & (M, 0) & - & (L, 0) \\
(V H, 0) & (V L, 0) & (V H, 0) & -
\end{array}\right) ; P_{e_{2}}=\left(\begin{array}{cccc}
- & (V L, 0) & (L, 0) & (V H, 0) \\
(H, 0) & - & (Q H, 0) & (T, 0) \\
(Q H, 0) & (L, 0) & - & (H, 0) \\
(V L, 0) & (N, 0) & (L, 0) & -
\end{array}\right)
$$




$$
P_{e_{3}}=\left(\begin{array}{cccc}
- & (V L, 0) & (M, 0) & (V H, 0) \\
(H, 0) & - & (Q H, 0) & (L, 0) \\
(M, 0) & (L, 0) & - & (T, 0) \\
(L, 0) & (H, 0) & (N, 0) & -
\end{array}\right) ; P_{e_{4}}=\left(\begin{array}{cccc}
- & (V H, 0) & (Q H, 0) & (M, 0) \\
(V L, 0) & - & (M, 0) & (V H, 0) \\
(L, 0) & (M, 0) & - & (V L, 0) \\
(M, 0) & (L, 0) & (Q H, 0) & -
\end{array}\right)
$$

\section{FIRST ROUND}

In the following, we show how to apply each step of the consensus model.

(i) Computing consensus degrees: Firstly, the similarity matrix for each pair of experts is computed, and so, the consensus matrix is obtained. Then, the consensus degrees on pairs of alternatives, alternatives and global relation are obtained from the consensus matrix.

(a) Similarity matrices:

$$
\begin{aligned}
& S M_{12}=\left(\begin{array}{cccc}
- & 0.50 & 0.50 & 0.37 \\
0.50 & - & 0.75 & 0.62 \\
0.50 & 0.75 & - & 0.62 \\
0.25 & 0.87 & 0.37 & -
\end{array}\right) ; S M_{13}=\left(\begin{array}{cccc}
- & 0.50 & 0.75 & 0.37 \\
0.50 & - & 0.75 & 0.62 \\
0.75 & 0.75 & - & 0.25 \\
0.37 & 0.50 & 0.12 & -
\end{array}\right) \\
& S M_{14}=\left(\begin{array}{cccc}
- & 0.75 & 1.00 & 0.75 \\
1.00 & - & 1.00 & 0.75 \\
1.00 & 1.00 & - & 0.87 \\
0.62 & 0.87 & 0.87 & -
\end{array}\right) ; S M_{23}=\left(\begin{array}{cccc}
- & 1.00 & 0.75 & 1.00 \\
1.00 & - & 1.00 & 0.25 \\
0.75 & 1.00 & - & 0.62 \\
0.87 & 0.37 & 0.75 & -
\end{array}\right) \\
& S M_{24}=\left(\begin{array}{cccc}
- & 0.25 & 0.50 & 0.62 \\
0.50 & - & 0.75 & 0.87 \\
0.50 & 0.75 & - & 0.50 \\
0.62 & 0.75 & 0.50 & -
\end{array}\right) ; S M_{34}=\left(\begin{array}{cccc}
- & 0.25 & 0.75 & 0.62 \\
0.50 & - & 0.75 & 0.37 \\
0.75 & 0.75 & - & 0.12 \\
0.75 & 0.62 & 0.75 & -
\end{array}\right)
\end{aligned}
$$

(b) Consensus matrix:

$$
C M=\left(\begin{array}{cccc}
- & 0.54 & 0.70 & 0.62 \\
0.66 & - & 0.83 & 0.58 \\
0.70 & 0.83 & - & 0.50 \\
0.58 & 0.66 & 0.56 & -
\end{array}\right)
$$

(c) Consensus degrees on pairs of alternatives. The element $(l, k)$ of $C M$ represents the consensus degrees on the pair of alternatives $\left(x_{l}, x_{k}\right)$.

(d) Consensus on alternatives:

$$
c a^{1}=0.62 \quad c a^{2}=0.69 \quad c a^{3}=0.67 \quad c a^{4}=0.60
$$

(e) Consensus on the relation:

$$
c r=0.65
$$

(ii) Controlling the consensus state: In this step of the consensus model, the global consensus value, $\mathrm{cr}$, is compared with the minimum consensus threshold, $\gamma$. In this example, we have decided to use the value, $\gamma=0.75$. Because $c r<$ $\gamma$, then it is concluded that there is no consensus amongst the experts, and consequently, the proximity measures are computed in order to support the experts on the necessary changes in their preferences in order to increase $\mathrm{cr}$. 
(iii) Feedback mechanism: In this step, the proximity measures are computed. To do so, firstly the collective unbalanced fuzzy linguistic preference relation is obtained by aggregating all individual preference relations. In this case, this is done using the $L O W A_{u n}$ operator and the linguistic quantifier most of defined as $Q(r)=r^{1 / 2}$, which applying $(2.8)$, generates the following weighting vector $\{0.5,0.20,0.16,0.14\}$.

$$
P_{e_{c}}=\left(\begin{array}{cccc}
- & (M, 0.40) & (H, 0.04) & (Q H,-0.36) \\
(M,-0.30) & - & (Q H, 0.46) & (Q H,-0.46) \\
(M, 0.20) & (M,-0.40) & - & (H, 0.22) \\
(H,-0.38) & (M,-0.49) & (H,-0.26) & -
\end{array}\right)
$$

(a) Computation of proximity measures.

i. Proximity matrices:

$$
\begin{aligned}
P M_{1} & =\left(\begin{array}{cccc}
- & 0.92 & 0.88 & 0.54 \\
0.66 & - & 0.70 & 0.93 \\
0.72 & 0.95 & - & 0.60 \\
0.83 & 0.68 & 0.72 & -
\end{array}\right) ; P M_{2}=\left(\begin{array}{cccc}
- & 0.57 & 0.62 & 0.83 \\
0.83 & - & 0.94 & 0.69 \\
0.65 & 0.80 & - & 0.97 \\
0.55 & 0.56 & 0.65 & -
\end{array}\right) \\
P M_{3} & =\left(\begin{array}{cccc}
- & 0.57 & 0.87 & 0.83 \\
0.83 & - & 0.94 & 0.56 \\
0.97 & 0.80 & - & 0.65 \\
0.67 & 0.81 & 0.41 & -
\end{array}\right) ; P M_{4}=\left(\begin{array}{cccc}
- & 0.67 & 0.88 & 0.80 \\
0.66 & - & 0.70 & 0.82 \\
0.72 & 0.95 & - & 0.47 \\
0.92 & 0.81 & 0.84 & -
\end{array}\right)
\end{aligned}
$$

ii. Proximity on pairs of alternatives for expert $e_{i}$ are given in $P M_{i}$.

iii. Proximity on alternatives: see Table 1.

Table 1. Proximity measures on alternatives.

\begin{tabular}{|c|c|c|c|}
\hline$x_{1}$ & $x_{2}$ & $x_{3}$ & $x_{4}$ \\
\hline$p a_{1}^{1}=0.78$ & $p a_{1}^{2}=0.76$ & $p a_{1}^{3}=0.75$ & $p a_{1}^{4}=0.74$ \\
\hline$p a_{2}^{1}=0.67$ & $p a_{2}^{2}=0.82$ & $p a_{2}^{3}=0.80$ & $p a_{2}^{4}=0.58$ \\
\hline$p a_{3}^{1}=0.75$ & $p a_{3}^{2}=0.77$ & $p a_{3}^{3}=0.80$ & $p a_{3}^{4}=0.63$ \\
\hline$p a_{4}^{1}=0.78$ & $p a_{4}^{2}=0.72$ & $p a_{4}^{3}=0.71$ & $p a_{4}^{4}=0.86$ \\
\hline
\end{tabular}

iv. Proximity on the relation:

$$
p r_{1}=0.76 \quad p r_{2}=0.72 \quad p r_{3}=0.74 \quad p r_{4}=0.77
$$

(b) Production of advice.

i. Identification rules.

(IR.1) Set of experts to change their preferences, $E X P C H$ :

$$
E X P C H=\left\{i \mid p r_{i}<0.75\right\}=\left\{e_{2}, e_{3}\right\}
$$

(IR.2) Set of alternatives whose assessments should be considered in the change process, $A L T_{i}$ :

$$
A L T_{i}=\left\{x_{l} \in X \mid c a^{l}<0.75 \wedge e_{i} \in E X P C H\right\}=\left\{x_{1}, x_{2}, x_{3}, x_{4}\right\}
$$


(IR.3) Set of pairs of alternatives whose associated assessments should change, $P A L T_{i}$ :

$P A L T_{2}=\left\{\left(x_{1}, x_{2}\right),\left(x_{1}, x_{3}\right),\left(x_{2}, x_{4}\right),\left(x_{3}, x_{1}\right),\left(x_{4}, x_{1}\right),\left(x_{4}, x_{2}\right),\left(x_{4}, x_{3}\right)\right\}$

which gives the following list of preference values:

$$
p_{2}^{12} \quad p_{2}^{13} \quad p_{2}^{24} \quad p_{2}^{31} \quad p_{2}^{41} \quad p_{2}^{42} \quad p_{2}^{43}
$$

and $P A L T_{3}=\left\{\left(x_{1}, x_{2}\right),\left(x_{2}, x_{4}\right),\left(x_{3}, x_{4}\right),\left(x_{4}, x_{1}\right),\left(x_{4}, x_{3}\right)\right\}$ which gives the following list of preference values:

$$
p_{3}^{12} p_{3}^{24} p_{3}^{34} p_{3}^{41} p_{3}^{43}
$$

ii. Direction rules.

Because $p_{2}^{12}<p_{c}^{12}, p_{2}^{13}<p_{c}^{13}, p_{2}^{41}<p_{c}^{41}, p_{2}^{42}<p_{c}^{42}, p_{2}^{43}<p_{c}^{43}$ and $p_{2}^{24}>p_{c}^{24}, p_{2}^{31}>p_{c}^{31}$ expert $e_{2}$ is advised to increase the assessment of the first five preference values and decrease the assessment of the last two preference values. For the same reason, because $p_{3}^{12}<p_{c}^{12}$, $p_{3}^{24}<p_{c}^{24}, p_{3}^{41}<p_{c}^{41}, p_{3}^{43}<p_{c}^{43}$ and $p_{3}^{34}>p_{c}^{34}$ expert $e_{3}$ is advised to increase the assessment of the first four preference values and decrease the assessment of the last one preference value.

\section{SECOND ROUND}

(i) Providing new preferences: In this example, we suppose that experts $e_{2}$ and $e_{3}$ follow the advice given, and thus, their new preferences are as follows:

$$
P_{e_{2}}=\left(\begin{array}{cccc}
- & (\mathbf{M}, \mathbf{0}) & (\mathbf{H}, \mathbf{0}) & (V H, 0) \\
(H, 0) & - & (Q H, 0) & (\mathbf{Q H}, \mathbf{0}) \\
(\mathbf{M}, \mathbf{0}) & (L, 0) & - & (H, 0) \\
(\mathbf{M}, \mathbf{0}) & (\mathbf{M}, \mathbf{0}) & (\mathbf{H}, \mathbf{0}) & -
\end{array}\right) ; P_{e_{3}}=\left(\begin{array}{cccc}
- & (\mathbf{M}, \mathbf{0}) & (M, 0) & (V H, 0) \\
(H, 0) & - & (Q H, 0) & (\mathbf{Q H}, \mathbf{0}) \\
(M, 0) & (L, 0) & - & (\mathbf{H}, \mathbf{0}) \\
(\mathbf{M}, \mathbf{0}) & (H, 0) & (\mathbf{H}, \mathbf{0}) & -
\end{array}\right)
$$

(ii) Computing consensus degrees.

(a) Similarity matrices:

$$
\begin{aligned}
& S M_{12}=\left(\begin{array}{cccc}
- & 0.87 & 0.87 & 0.37 \\
0.50 & - & 0.75 & 0.87 \\
0.75 & 0.75 & - & 0.62 \\
0.62 & 0.62 & 0.75 & -
\end{array}\right) ; S M_{13}=\left(\begin{array}{cccc}
- & 0.87 & 0.75 & 0.37 \\
0.50 & - & 0.75 & 0.87 \\
0.75 & 0.75 & - & 0.62 \\
0.62 & 0.50 & 0.75 & -
\end{array}\right) \\
& S M_{14}=\left(\begin{array}{cccc}
- & 0.75 & 1.00 & 0.75 \\
1.00 & - & 1.00 & 0.75 \\
1.00 & 1.00 & - & 0.87 \\
0.62 & 0.87 & 0.87 & -
\end{array}\right) ; S M_{23}=\left(\begin{array}{cccc}
- & 1.00 & 0.87 & 1.00 \\
1.00 & - & 1.00 & 1.00 \\
1.00 & 1.00 & - & 1.00 \\
1.00 & 0.87 & 1.00 & -
\end{array}\right) \\
& S M_{24}=\left(\begin{array}{cccc}
- & 0.62 & 0.87 & 0.62 \\
0.50 & - & 0.75 & 0.87 \\
0.75 & 0.75 & - & 0.50 \\
1.00 & 0.75 & 0.87 & -
\end{array}\right) ; S M_{34}=\left(\begin{array}{ccccc}
- & 0.62 & 0.75 & 0.62 \\
0.50 & - & 0.75 & 0.87 \\
0.75 & 0.75 & - & 0.50 \\
1.00 & 0.62 & 0.87 & -
\end{array}\right)
\end{aligned}
$$


(b) Consensus matrix:

$$
C M=\left(\begin{array}{cccc}
- & 0.78 & 0.85 & 0.63 \\
0.66 & - & 0.83 & 0.87 \\
0.83 & 0.83 & - & 0.68 \\
0.81 & 0.71 & 0.85 & -
\end{array}\right)
$$

(c) Consensus degrees on pairs of alternatives. The element $(l, k)$ of $C M$ represents the consensus degrees on the pair of alternatives $\left(x_{l}, x_{k}\right)$.

(d) Consensus on alternatives:

$$
c a^{1}=0.75 \quad c a^{2}=0.79 \quad c a^{3}=0.78 \quad c a^{4}=0.79
$$

(e) Consensus on the relation:

$$
c r=0.78
$$

(iii) Controlling the consensus state: As we can observe, the changes in the preference values introduced result in an increasing of the global consensus from 0.65 to 0.78 . The minimum consensus threshold is reached, $c r=0.78>$ $\gamma=0.75$, and, therefore, the consensus model would stop and the selection process would be applied to obtain the final solution of consensus.

\section{Concluding Remarks}

In this paper we have proposed a consensus model for GDM problems with unbalanced fuzzy linguistic information. There are two main advantages provided by this consensus model: it is able to manage consensus process in GDM problems with unbalanced fuzzy linguistic information overcoming the problems of unbalanced fuzzy linguistic approach defined in, ${ }^{1}$ and it is able to support the consensus process automatically, without moderator, traditionally present in the consensus process.

In the future we think to extend our consensus model to deal with situations under incomplete information ${ }^{5,10}$ and to build Web-based distributed consensus support systems ${ }^{35}$.

\section{Acknowledgments}

This paper has been developed with the financing of SAINFOWEB project (00602) and FUZZYLING project (TIN2007-61079).

\section{References}

1. E. Herrera-Viedma and A.G. López-Herrera, A model of information retrieval system with unbalanced fuzzy linguistic information, International Journal of Intelligent Systems 22:11 (2007) 1197-1214.

2. S.J. Chen and C.L. Hwang, Fuzzy multiple attributive decision making: Theory and its applications (Springer, Berlín, 1992). 
3. F. Herrera, E. Herrera-Viedma and J.L. Verdegay, A model of consensus in group decision making under linguistic assessments, Fuzzy Sets and Systems 78 (1996) 7387.

4. E. Herrera-Viedma, F. Herrera and F. Chiclana, A consensus model for multiperson decision making with different preference structures, IEEE Transactions on Systems, Man and Cybernetics-Part A: Systems and Humans 32:3 (2002) 394-402.

5. E. Herrera-Viedma, F. Chiclana, F. Herrera and S. Alonso, A group decision-making model with incomplete fuzzy preference relations based on additive consistency, IEEE Transactions on Systems, Man and Cybernetics-Part B: Cybernetics 37:1 (2007) 176189.

6. J. Kacprzyk, M. Fedrizzi and H. Nurmi, Group decision making and consensus under fuzzy preferences and fuzzy majority, Fuzzy Sets and Systems 49 (1992) 21-31.

7. J. Kacprzyk, H. Nurmi and M. Fedrizzi, Consensus under fuzziness (Kluwer Academic Publishers, Boston, 1997).

8. T. Shigenobu, T. Yoshino and J. Munemori, Evaluation and application of creativity collabotarion support system GUGEN DX II for consensus-building among users, International Journal of Information Technology \& Decision Making 6:3 (2007) 475490.

9. E. Herrera-Viedma, L. Martínez, F. Mata and F. Chiclana, A consensus support system model for group decision-making problems with multi-granular linguistic preference relations, IEEE Transaction on Fuzzy Systems 13:5 (2005) 644-658.

10. E. Herrera-Viedma, S. Alonso, F. Herrera and F. Chiclana, A consensus model for group decision making with incomplete fuzzy preference relations, IEEE Transactions on Fuzzy Systems 15:5 (2007) 863-877.

11. F. Herrera, E. Herrera-Viedma and J.L. Verdegay, A linguistic decision process in group decision making, Group Decision and Negotiation 5 (1996) 165-176.

12. F. Herrera, E. Herrera-Viedma and J.L. Verdegay, Choice processes for nonhomogeneous group decision making in linguistic setting, Fuzzy Sets and Systems 94 (1998) 287-308.

13. E. Herrera-Viedma, G. Pasi, A.G. López-Herrera and C. Porcel, Evaluating the information quality of Web sites: A methodology based on fuzzy computing with words, Journal of the American Society for Information Science and Technology 57:4 (2006) $538-549$.

14. J. Ma, D. Ruan, Y. Xu and G. Zhang, A fuzzy-set approach to treat determinacy and consistency of linguistic terms in multi-criteria decision making, International Journal of Approximate Reasoning 44:2 (2007) 165-181.

15. Z.S. Xu, An approach to group decision making based on incomplete linguistic preference relations, International Journal of Information Technology \& Decision Making 4:1 (2005) 153-160.

16. Z.S. Xu, An approach to pure linguistic multiple attribute decision making under uncertainty, International Journal of Information Technology \& Decision Making 4:2 (2005) 197-206.

17. Z.S. Xu, A method for multiple attribute decision making with incomplete weight information in linguistic setting, Knowledge-Based Systems 20:8 (2007) 719-725.

18. L.A. Zadeh, The concept of a linguistic variable and its applications to approximate reasoning, Part I, Information Sciencies 8 (1975) 199-249.

19. L.A. Zadeh, The concept of a linguistic variable and its applications to approximate reasoning, Part II, Information Sciencies 8 (1975) 301-357.

20. L.A. Zadeh, The concept of a linguistic variable and its applications to approximate reasoning, Part III, Information Sciencies 9 (1975) 43-80. 
21. E. Levrat, A. Voisin, S. Bombardier and J. Bremont, Subjective evaluation of car seat comfort with fuzzy set techniques, International Journal of Intelligent Systems $\mathbf{1 2}$ (1997) 891-913.

22. V. Torra, Aggregation of linguistic labels when semantics is based on antonyms, International Journal of Intelligent Systems 16 (2001) 513-524.

23. F. Herrera and L. Martínez, A 2-tuple fuzzy linguistic representation model for computing with words, IEEE Transactions on Fuzzy Systems 8:6 (2000) 746-752.

24. F. Herrera and L. Martínez, A model based on linguistic 2-tuples for dealing with multigranularity hierarchical linguistic contexts in multiexpert decision-making, IEEE Transactions on Systems, Man and Cybernetics-Part B: Cybernetics 31:2 (2001) 227234.

25. F. Herrera, E. Herrera-Viedma and J.L. Verdegay, Direct approach processes in group decision making using linguistic OWA operators, Fuzzy Sets and Systems 79 (1996) $175-190$

26. F. Herrera and E. Herrera-Viedma, Linguistic decision analysis: steps for solving decisions problems under linguistic information, Fuzzy Sets and Systems 115 (2000) $67-82$.

27. E. Herrera-Viedma, Modeling the retrieval process of an information retrieval system using an ordinal fuzzy linguistic approach, Journal of the American Society for Information Science and Technology 52:6 (2001) 460-475.

28. E. Herrera-Viedma, An information retrieval system with ordinal linguistic weighted queries based on two weighting elements, International Journal of Uncertainty, Fuzziness and Knowledge-Based Systems 9 (2001) 77-88.

29. S.L. Chang, R.C. Wang and S.Y. Wang, Applying a direct multigranularity linguistic and strategy-oriented aggregation approach on the assessment of supply performance, European Journal of Operational Research 177:2 (2007) 1013-1025.

30. F. Herrera, E. Herrera-Viedma and L. Martínez, A fusion approach for managing multi-granularity linguistic term sets in decision making, Fuzzy Sets and Systems 114 (2000) 43-58.

31. R.R. Yager, On ordered weighted averaging aggregation operators in multicriteria decision making, IEEE Transactions on Systems, Man and Cybernetics-Part B: Cybernetics 10:1 (1988) 183-190.

32. L.A. Zadeh, A computational approach to fuzzy quantifiers in natural language, Computation \& Mathematics with Applications 9 (1983) 149-184.

33. F. Herrera, E. Herrera-Viedma and J.L. Verdegay, A rational consensus model in group decision making using linguistic assessments, Fuzzy Sets and Systems 88 (1997) 31-49.

34. S. Zadrozny, An approach to the consensus reaching support in fuzzy environment, in Consensus under fuzziness, eds. J. Kacprzyk, H. Nurmi and M. Fedrizzi (Kluwer Academic Publishers, Massachusetts, 1997), pp. 83-109.

35. M. Tavana and D.T. Kennedy, N-Site: a distributed consensus building and negotiation support system, International Journal of Information Technology \& Decision Making 5:1 (2006) 123-154. 